PROCEEDINGS OF THE

AMERICAN MATHEMATICAL SOCIETY

Volume 141, Number 1, January 2013, Pages 313-324

S 0002-9939(2012)11294-8

Article electronically published on May 3, 2012

\title{
LIMIT OF QUASILOCAL MASS INTEGRALS IN ASYMPTOTICALLY HYPERBOLIC MANIFOLDS
}

\author{
KWOK-KUN KWONG AND LUEN-FAI TAM
}

(Communicated by Jianguo Cao)

\begin{abstract}
In this paper, we will show that the limit of some quasilocal mass integrals of the coordinate spheres in an asymptotically hyperbolic (AH) manifold is the mass integral of the AH manifold. This is the analogue of the well-known result that the limit of the Brown-York mass of coordinate spheres is the ADM mass in an asymptotically flat manifold.
\end{abstract}

\section{INTRODUCTION}

It is well known that a good definition of quasilocal mass should satisfy positivity. It should also be such that the quasilocal mass of coordinate spheres should converge to the ADM mass of an asymptotically flat manifold. Under reasonable assumptions, the Brown-York quasilocal mass satisfies both conditions by [10, 8, 5, 12, 4, In this work, we want to study the behavior of some quasilocal mass quantities of coordinate spheres in asymptotically hyperbolic $(\mathrm{AH})$ manifolds. As for positivity, M.-T. Wang and Yau [15] proved the following. Let $(\Omega, g)$ be a three-dimensional compact manifold with scalar curvature $R \geq-6$ with smooth boundary $\Sigma$ which is homeomorphic to the standard sphere $\mathbb{S}^{2}$ such that the mean curvature $H$ of $\Sigma$ is positive and the Gaussian curvature of $\Sigma$ is larger than -1 . By [9, $\Sigma$ can be isometrically embedded into the hyperbolic space $\mathbb{H}^{3}$ with positive mean curvature $H_{0}$, and the embedding is unique up to an isometry of $\mathbb{H}^{3}$. Consider $\mathbb{H}^{3}$ as the hyperboloid in $\mathbb{R}^{3,1}$ :

$$
\mathbb{H}^{3}=\left\{\left(x^{0}, x^{1}, x^{2}, x^{3}\right) \in \mathbb{R}^{3,1}:\left(x^{0}\right)^{2}-\sum_{i=1}^{3}\left(x^{i}\right)^{2}=1, x^{0}>0\right\} .
$$

Wang and Yau proved that under these assumptions on $\Omega$ and $\Sigma$, there is a future timelike vector $W$ such that $\int_{\Sigma}\left(H_{0}-H\right) W$ is a future directed nonspacelike vector, where $W$ is obtained by solving a backward parabolic equation with prescribed data at infinity. $W$ is not very explicit. In [11, Shi and the second author proved that if $B_{o}\left(R_{1}\right)$ and $B_{o}\left(R_{2}\right)$ are two geodesic balls in $\mathbb{H}^{3}$ such that $B_{o}\left(R_{1}\right) \subset D \subset B_{o}\left(R_{2}\right)$ with $\partial D=\Sigma$, where $o=(1,0,0,0) \in \mathbb{H}^{3} \subset \mathbb{R}^{3,1}$, then the result of Wang-Yau is

Received by the editors March 9, 2011 and, in revised form, June 7, 2011.

2010 Mathematics Subject Classification. Primary 53C20; Secondary 83C99.

Key words and phrases. Quasilocal mass integral, asymptotically hyperbolic manifolds, isometric embedding.

This research was partially supported by Hong Kong RGC General Research Fund \#CUHK 403108.

(C)2012 American Mathematical Society Reverts to public domain 28 years from publication 
true for $W=W_{1}\left(x^{0}, x^{1}, x^{2}, x^{3}\right)=\left(\alpha x^{0}, x^{1}, x^{2}, x^{3}\right)$ with some $\alpha>1$, where

$$
\alpha=\operatorname{coth} R_{1}+\frac{1}{\sinh R_{1}}\left(\frac{\sinh ^{2} R_{2}}{\sinh ^{2} R_{1}}-1\right)^{\frac{1}{2}} .
$$

Therefore it is interesting to see if $\int_{\Sigma}\left(H_{0}-H\right) W_{1}$ for coordinate spheres in an $\mathrm{AH}$ manifold will converge to the mass integral (see below) of the manifold. Instead, in this work we want to study the limiting behavior of the quasilocal mass integrals $\int_{\Sigma}\left(H_{0}-H\right) X$ of coordinate spheres in an AH manifold, where $X$ is the position vector, because this expression is simpler, and by the estimates in sections 2 to 4 , it is easy to see that $\int_{\Sigma}\left(H_{0}-H\right)\left(W_{1}-X\right)$ will tend to zero. In fact, it is an open question whether the results in [15, 11] are still true if $W=X$.

We will follow X. D. Wang [14 to define asymptotically hyperbolic manifolds as follows; all manifolds are assumed to be connected and orientable:

Definition 1.1. A complete noncompact Riemannian manifold $\left(M^{n}, g\right)$ is said to be asymptotically hyperbolic (AH) if $M$ is the interior of a compact manifold $\bar{M}$ with boundary $\partial \bar{M}$ such that:

(i) there is a smooth function $r$ on $\bar{M}$ with $r>0$ on $M$ and $r=0$ on $\partial \bar{M}$ such that $\bar{g}=r^{2} g$ extends as a smooth Riemannian metric on $\bar{M}$;

(ii) $|d r|_{\bar{g}}=1$ on $\partial \bar{M}$;

(iii) $\partial \bar{M}$ is the standard unit sphere $\mathbb{S}^{n-1}$;

(iv) on a collar neighborhood of $\partial \bar{M}$,

$$
g=\sinh ^{-2}(r)\left(d r^{2}+g_{r}\right),
$$

$g_{r}$ being an $r$-dependent family of metrics on $\mathbb{S}^{n-1}$ such that

$$
g_{r}=g_{0}+\frac{r^{n}}{n} h+e,
$$

where $g_{0}$ is the standard metric, $h$ is a smooth symmetric 2-tensor on $\mathbb{S}^{n-1}$ and $e$ is of order $O\left(r^{n+1}\right)$, and the asymptotic expansion can be differentiated four times.

Note that the definition is not as general as that in [2]; see also [16]. The following positive mass theorem was proved by X. D. Wang [14] (see also [1, 2, 16]).

Theorem 1.2 ([14, Theorem 2.5]). If $\left(M^{n}, g\right)$ is spin, asymptotically hyperbolic and the scalar curvature $R \geq-n(n-1)$, then

$$
\int_{\mathbb{S}^{n-1}} \operatorname{tr}_{g_{0}}(h) d \mu_{g_{0}} \geq\left|\int_{\mathbb{S}^{n-1}} \operatorname{tr}_{g_{0}}(h) x d \mu_{g_{0}}\right| .
$$

Moreover equality holds if and only if $(M, g)$ is isometric to the hyperbolic space $\mathbb{H}^{n}$.

We only consider the case that $n=3$. The theorem implies that if $M$ is not isometric to the hyperbolic space, then the vector

$$
\Upsilon=\left(\int_{\mathbb{S}^{n-1}} \operatorname{tr}_{g_{0}}(h) d \mu_{g_{0}}, \int_{\mathbb{S}^{n-1}} \operatorname{tr}_{g_{0}}(h) x d \mu_{g_{0}}\right)
$$

is a future-directed timelike vector in $\mathbb{R}^{3,1}$, the Minkowski space. We may consider $\Upsilon$ as the mass integral for the AH manifold. 
In this work, we consider $\mathrm{AH}$ manifolds with the following condition (with the notation as in Definition 1.1):

Assumption A. $\nabla_{\mathbb{S}^{n-1}} e, \nabla_{\mathbb{S}^{n-1}}^{2} e, \nabla_{\mathbb{S}^{n-1}}^{3} e, \nabla_{\mathbb{S}^{n-1}}^{4} e$ with respect to $g_{0}$ and $\frac{\partial e}{\partial r}$ are of order $O\left(r^{n}\right)$.

Let $S_{a}=\{r=a\} \subset(M, g)$ and let $H$ be its mean curvature. We identify $S_{r}$ as the standard sphere $\mathbb{S}^{2}$ with metric $\gamma_{r}$ induced from $g$. Then for $r$ small, the Gaussian curvature of $\left(S_{r}, \gamma_{r}\right)$ is positive. Hence $\left(S_{r}, \gamma_{r}\right)$ can be isometrically embedded in $\mathbb{H}^{3}$. Let $X^{(r)}$ be an embedding such that $o$ is the center the largest geodesic sphere contained in the interior of $X^{(r)}\left(S_{r}\right)$ (or a smallest geodesic sphere containing $X^{(r)}\left(S_{r}\right)$ in its interior).

Our main result is the following:

Theorem 1.3. Let $(M, g)$ be a three-dimensional asymptotically hyperbolic manifold satisfying Assumption A. Then there are isometries $\iota_{r}$ of $\mathbb{H}^{3}$ fixing o such that

$$
\lim _{r \rightarrow 0} \int_{S_{r}}\left(H_{0}-H\right) \iota_{r} \circ X^{(r)} d \mu_{\gamma_{r}}=\frac{1}{2}\left(\int_{\mathbb{S}^{2}} \operatorname{tr}_{g_{0}}(h) d \mu_{g_{0}}, \int_{\mathbb{S}^{2}} \operatorname{tr}_{g_{0}}(h) x d \mu_{g_{0}}\right)
$$

where $H_{0}$ is the mean curvature of $X^{(r)}\left(S_{r}\right)$ in $\mathbb{H}^{3}$.

By applying Theorem 1.2 to our result, if the scalar curvature of $M$ is no less than -6 , then for $r$ small enough,

$$
\int_{S_{r}}\left(H_{0}-H\right) \iota_{r} \circ X^{(r)} d \mu_{\gamma_{r}}
$$

is either zero or is future-directed timelike. This improves the result in [1] for this particular case.

This paper is organized as follows. In Section 2, we will establish some estimates for some intrinsic and extrinsic curvatures of $S_{r}$ and its embedding in the hyperbolic space. In Section 3, we will describe some basic results in hyperbolic geometry concerning the radii of the smallest geodesic sphere enclosing a given convex surface and of the largest geodesic sphere enclosed by it. In Section 4 we will normalize the isometric embedding of $S_{r}$ into the hyperbolic space so that the image of the isometric embedding of $S_{r}$ is close to a geodesic sphere in the hyperbolic space. We then prove the main result in Section 5 ,

\section{Curvature estimates}

In this section, we always assume that $\left(M^{3}, g\right)$ is a three-dimensional AH manifold such that Assumption A is satisfied. Using the notation in Definition 1.1. let $S_{a}=\{r=a\} \subset M$. We will obtain some curvature estimates for $S_{r}$, which will be used in the proof of the main result. First we will estimate the intrinsic scalar curvature $R$, which is twice the Gaussian curvature of $S_{r}$ with the metric $\gamma_{r}$ induced by $g$.

Lemma 2.1. The scalar curvature $R$ of $S_{r}$ with respect to the induced metric from $g$ is given by

$$
R=2 \sinh ^{2} r+O\left(r^{5}\right) .
$$


Proof. Recall that $g_{r}=g_{0}+\frac{r^{3}}{3} h+e$. Then $\gamma_{r}=\sinh ^{-2}(r) g_{r}$ is the induced metric on $S_{r}$ from $g$. Let $R$ and $\tilde{R}$ be the scalar curvatures of $S_{r}$ with respect to the metrics $\gamma_{r}$ and $g_{r}$, respectively. It is easy to see that $R=\sinh ^{2}(r) \tilde{R}$. We claim that

$$
\tilde{R}=2+O\left(r^{3}\right) .
$$

The result immediately follows from this claim.

To show this, let $\left\{y^{i}\right\}_{i=1}^{2}$ be the local coordinates on the lower hemisphere (say) of $\mathbb{S}^{2}$ induced by the stereographic projection from the north pole to the plane. Let $\tilde{g}_{i j}=g_{r}\left(\frac{\partial}{\partial y^{2}}, \frac{\partial}{\partial y^{j}}\right), g_{i j}=g_{0}\left(\frac{\partial}{\partial y^{2}}, \frac{\partial}{\partial y^{j}}\right)$ and $\tilde{\Gamma}_{i j}^{k}, \Gamma_{i j}^{k}$ be the Christoffel symbols with respect to $\tilde{g}_{i j}$ and $g_{i j}$ respectively. Let $\tilde{g}^{i j}$ and $g^{i j}$ be the inverses of $\tilde{g}_{i j}$ and $g_{i j}$ respectively. Then

$\begin{cases}\tilde{R}=\sum_{j, k, l} \tilde{g}^{j k} \tilde{R}_{l j k}^{l}, & \text { where } \tilde{R}_{i j k}^{l}=\partial_{i} \tilde{\Gamma}_{j k}^{l}-\partial_{j} \tilde{\Gamma}_{k i}^{l}+\sum_{p} \tilde{\Gamma}_{j k}^{p} \tilde{\Gamma}_{i p}^{l}-\sum_{p} \tilde{\Gamma}_{i k}^{p} \tilde{\Gamma}_{j p}^{l}, \\ R=2=\sum_{j, k, l} g^{j k} R_{l j k}^{l}, & \text { where } R_{i j k}^{l}=\partial_{i} \Gamma_{j k}^{l}-\partial_{j} \Gamma_{k i}^{l}+\sum_{p} \Gamma_{j k}^{p} \Gamma_{i p}^{l}-\sum_{p} \Gamma_{i k}^{p} \Gamma_{j p}^{l} .\end{cases}$ Assumption A implies that

$$
\left|\tilde{g}_{i j}-g_{i j}\right|=O\left(r^{3}\right),\left|\tilde{g}_{i j, k}-g_{i j, k}\right|=O\left(r^{3}\right) \text { and }\left|\tilde{g}_{i j, k l}-g_{i j, k l}\right|=O\left(r^{3}\right),
$$

where $g_{i j, k}=\frac{\partial g_{i j}}{\partial y^{k}}$, etc. Hence

$$
\tilde{\Gamma}_{i j}^{k}-\Gamma_{i j}^{k}=O\left(r^{3}\right) \text { and } \partial_{i} \tilde{\Gamma}_{j k}^{l}-\partial_{i} \Gamma_{j k}^{l}=O\left(r^{3}\right) .
$$

In view of (2.2), these imply that $\tilde{R}_{i j k}^{l}-R_{i j k}^{l}=O\left(r^{3}\right)$ and so $\tilde{R}-2=O\left(r^{3}\right)$. Hence (2.1) is true. This completes the proof.

Next, we estimate the mean curvature $H$ of $S_{r}$ with respect to $g$.

Lemma 2.2. If $(M, g)$ is asymptotically hyperbolic satisfying Assumption A, then the mean curvature of $S_{r}$ is

$$
H=2 \cosh r-\frac{1}{2} r^{3} \operatorname{tr}_{g_{0}} h+O\left(r^{4}\right) .
$$

Proof. Let $\left\{e_{j}\right\}_{j=1}^{2}$ be a local orthonormal frame on $\left(\mathbb{S}^{2}, g_{0}\right)$. The outer unit normal of $S_{r}$ is $\nu=-\sinh r \frac{\partial}{\partial r}$. Denote $g\left(e_{i}, e_{j}\right)$ by $g_{i j}$ and $g_{r}\left(e_{i}, e_{j}\right)$ by $\sigma_{i j}$. Then

$$
\begin{aligned}
H=\nu\left(\log \sqrt{\operatorname{det}\left(g_{i j}\right)}\right) & =-\sinh r \frac{\partial}{\partial r}\left(\log \left(\sinh ^{-2} r \sqrt{\operatorname{det}\left(\sigma_{i j}\right)}\right)\right) \\
& =2 \cosh r-\frac{1}{2} \frac{\sinh r}{\sqrt{\operatorname{det}\left(\sigma_{i j}\right)}} \frac{\partial}{\partial r} \operatorname{det}\left(\sigma_{i j}\right) .
\end{aligned}
$$

It is easy to see that $\operatorname{det}\left(\sigma_{i j}\right)=1+\frac{r^{3}}{3} \operatorname{tr}_{g_{0}} h+O\left(r^{4}\right)$ and by the condition $\frac{\partial e}{\partial r}=O\left(r^{3}\right)$, that $\frac{\partial}{\partial r} \operatorname{det}\left(\sigma_{i j}\right)=r^{2} \operatorname{tr}_{g_{0}} h+O\left(r^{3}\right)$. Combining these with the above calculation, the result follows.

By Lemma 2.1 for sufficiently small $r$, the Gaussian curvature $K$ of $\left(S_{r}, \gamma_{r}\right)$ is positive. Hence $\left(S_{r}, \gamma_{r}\right)$ can be isometrically embedded into $\mathbb{H}^{3}$, which is unique up to an isometry in $\mathbb{H}^{3}$ by the results of Pogorelov 9 . Moreover, by the Gauss equation, for an orthonormal frame in $S_{r}$,

$$
-1+\chi_{11} \chi_{22}-\chi_{12}^{2}=K>0 .
$$

Hence the embedded surface, which will be denoted by $\Sigma_{r}$, is strictly convex. Let $H_{0}$ be the mean curvature of $\Sigma_{r}$. We want to estimate $H_{0}$ and compare it with $H$. 
To estimate $H_{0}$, we will generalize a result on convex compact hypersurfaces in $\mathbb{R}^{n}$ of Li-Weinstein [7, Theorem 2] to compact hypersurfaces in $\mathbb{H}^{n}$.

Lemma 2.3. Suppose $\Sigma$ is a closed convex hypersurface in $\mathbb{H}^{n}, n \geq 3$. If the scalar curvature $R$ of $\Sigma$ satisfies $R+(n-2)(n-3)>0$, then its mean curvature $H_{0}$ satisfies the inequality

$$
H_{0}^{2} \leq \max _{\Sigma}\left(\frac{2 \hat{R}^{2}-2(n-1) \hat{R}-\Delta R}{R+(n-2)(n-3)}\right)
$$

where $\hat{R}=R+(n-1)(n-2)$ and $\Delta$ is the Laplacian on $\Sigma$.

Proof. We basically follow the ideas from [7. Let $\chi$ be the second fundamental form of $\Sigma \subset \mathbb{H}^{n}$. Let $p \in \Sigma$ be such that $H_{0}(p)=\max _{\Sigma} H_{0}$. Let $\left\{x^{j}\right\}_{j=1}^{n-1}$ be normal coordinates of $\Sigma$ around $p$ so that $\chi_{i j}=\lambda_{i} \delta_{i j}$ at $p$. Then at $p, H_{0 ; i j}$ is negative semi-definite. Here we use $S_{; k}$ to denote the covariant derivative of $S$ on $\Sigma$ with respect to the induced metric. Since $\left(\chi_{i j}\right) \geq 0$ and $\left(H_{0 ; i j}\right) \leq 0$, at $p$ we have

$$
H_{0} \Delta H_{0} \leq \chi^{i j} H_{0 ; i j}=\sum_{i} \lambda_{i} H_{0 ; i i}
$$

Here and below, summations will be from 1 to $n-1$. Since $\mathbb{H}^{n}$ has constant curvature, the Codazzi equation implies that

$$
\chi_{i j ; k}-\chi_{i k ; j}=0 .
$$

By the Gauss equation, we have

$$
R+(n-1)(n-2)=H_{0}^{2}-|\chi|^{2} .
$$

Let $R_{i j k l}$ be the intrinsic curvature tensor of $\Sigma$. At $p$,

$$
\begin{aligned}
\Delta R & =2 H_{0} \Delta H_{0}+2\left|\nabla H_{0}\right|^{2}-2|\nabla \chi|^{2}-2 \sum_{i, k} \lambda_{i} \chi_{i i ; k k} \\
& \leq 2 \sum_{i, k} \lambda_{i}\left(\chi_{k k ; i i}-\chi_{i i ; k k}\right) \quad\left(\text { by }(\underline{2.4}) \text { and } \nabla H_{0}=0\right) \\
& =2 \sum_{i, k} \lambda_{i}\left(\chi_{k k ; i i}-\chi_{k i ; i k}\right) \quad(\text { by (2.5) }) \\
& \left.=2 \sum_{i, k, m} \lambda_{i}\left(R_{k i k m} \chi_{m i}+R_{k i i m} \chi_{k m}\right) \quad \text { (by the Ricci identity and (2.5) }\right) \\
& =2 \sum_{i, k} R_{k i i k}\left(-\lambda_{i}^{2}+\lambda_{i} \lambda_{k}\right) \\
& =2 \sum_{i, k}\left(-1+\lambda_{k} \lambda_{i}\right)\left(-\lambda_{i}^{2}+\lambda_{i} \lambda_{k}\right) \quad(\text { by the Gauss equation) } \\
& =2\left((n-1)|\chi|^{2}-H_{0} \sum_{i} \lambda_{i}^{3}-H_{0}^{2}+|\chi|^{4}\right) .
\end{aligned}
$$

By [7, Lemma 2], since $\lambda_{i}>0$,

$$
-2 \sum_{i} \lambda_{i}^{3} \leq\left(\sum_{i} \lambda_{i}\right)^{3}-3\left(\sum_{i} \lambda_{i}^{2}\right)\left(\sum_{i} \lambda_{i}\right)=H_{0}^{3}-3|\chi|^{2} H_{0} .
$$


Plugging this into the above and using (2.6), at $p$,

$$
\begin{aligned}
\Delta R & \leq 2(n-1)|\chi|^{2}+3 \hat{R} H_{0}^{2}-2 H_{0}^{4}+2|\chi|^{4}-2 H_{0}^{2} \\
& =2(n-1)\left(H_{0}^{2}-\hat{R}\right)+3 \hat{R} H_{0}^{2}-2 H_{0}^{4}+2\left(H_{0}^{2}-\hat{R}\right)^{2}-2 H_{0}^{2} \\
& =-(\hat{R}-2(n-2)) H_{0}^{2}-2(n-1) \hat{R}+2 \hat{R}^{2} .
\end{aligned}
$$

From this it is easy to see that the lemma is true.

Applying the previous lemma to $\Sigma_{r}$, which is the embedded image of $\left(S_{r}, \gamma_{r}\right)$, we have:

Corollary 2.4. With the same assumptions and notation as in Lemma 2.1, for sufficiently small $r$, the mean curvature $H_{0}$ of $\Sigma_{r}$ in $\mathbb{H}^{3}$ satisfies

$$
H_{0}^{2} \leq \max _{S_{r}}\left(2 R+4-\frac{\Delta R}{R}\right)
$$

where $\Delta$ is the Laplacian on $S_{r}$ under the induced metric, $R=2 K$ and $K$ is the Gaussian curvature of $S_{r}$.

We now estimate $H_{0}$.

Lemma 2.5. The mean curvature $H_{0}$ of $\Sigma_{r}$ in $\mathbb{H}^{3}$ is given by

$$
H_{0}=2 \cosh r+O\left(r^{5}\right) \text {. }
$$

Proof. By the Gauss equation, $2 \hat{R} \leq \hat{R}+|\chi|^{2}=H_{0}^{2}$, where $\hat{R}=R+2$ and $\chi$ is the second fundamental form of $\Sigma_{r}$. By Lemma 2.1 and Corollary 2.4, we have

$$
4 \cosh ^{2} r+O\left(r^{5}\right) \leq H_{0}^{2} \leq 4 \cosh ^{2} r+\max _{S_{r}}\left|\frac{\Delta R}{R}\right|+O\left(r^{5}\right) .
$$

The proof will be complete if we can show that $\frac{\Delta R}{R}=O\left(r^{5}\right)$. The proof is analogous to that of Lemma 2.1. Using the notation as in the proof of Lemma 2.1. it is easy to see that

$$
\frac{\Delta R}{R}=\frac{\sinh ^{4} r}{R} \Delta_{g_{r}} \tilde{R}
$$

where $\tilde{R}$ is the scalar curvature with respect to $g_{r}$. Using Assumption A, we have

$$
\left|\partial^{(k)} \tilde{\Gamma}_{i j}^{l}-\partial^{(k)} \Gamma_{i j}^{l}\right|=O\left(r^{3}\right) \text { for } k=0,1,2,3,
$$

with respect to the coordinates $\left\{y^{i}\right\}_{i=1}^{2}$. Together with (2.2), we conclude that $\partial_{i} \tilde{R}-\partial_{i} R=O\left(r^{3}\right)$ and $\partial_{i j}^{2} \tilde{R}-\partial_{i j}^{2} R=O\left(r^{3}\right)$. Hence

$$
\Delta_{g_{r}} \tilde{R}-\Delta_{g_{0}} R_{0}=O\left(r^{3}\right) .
$$

As $R_{0}=2$ is a constant, by (2.7) and Lemma 2.1] the result follows.

Combining Lemma 2.2 and Lemma 2.5, we obtain

Corollary 2.6. On $S_{r}$, we have $H_{0}-H=\frac{1}{2} r^{3} \operatorname{tr}_{g_{0}} h+O\left(r^{4}\right)$. 


\section{INSCRIBED AND CIRCUMSCRIBED GEODESIC SPHERES}

It is well known that a compact convex hypersurface $\Sigma$ in $\mathbb{R}^{n}$ can contain and be contained in spheres with radius depending only on the upper and lower bounds of principal curvatures $\lambda_{i}$. In this section, we will describe the corresponding results in $\mathbb{H}^{n}$, which will be used later. We will sketch the proofs for the sake of completeness whenever we could not locate a reference. We only consider the case $n=3$. The general case is similar. The following is a direct consequence of a result of Ralph Howard [6, Theorem 4.5]. We thank him for this information.

Proposition 3.1. Let $\Sigma$ be a compact convex surface in $\mathbb{H}^{3}$ and suppose

$$
\operatorname{coth} b=\max _{x \in \Sigma, i=1,2} \lambda_{i}(x) \geq \min _{x \in \Sigma, i=1,2} \lambda_{i}(x)>1 \text {. }
$$

Then there is a geodesic sphere of radius $b$ which is contained in the interior of $\Sigma$.

Proof. By [6, Theorem 4.5], since $\lambda_{i}>1$ on $\Sigma$, the largest radius of geodesic balls which can roll inside $\Sigma$ is equal to the focal distance of $\Sigma$. It is not hard to see that the focal distance of $\Sigma$ in $\mathbb{H}^{3}$ is equal to

$$
\min _{x \in \Sigma}\left\{\rho: \operatorname{coth} \rho=\max \left\{\lambda_{1}(x), \lambda_{2}(x)\right\}\right\} .
$$

This can be seen by considering the $\Sigma$-Jacobi field along the inward-pointing geodesics perpendicular to $\Sigma$; see for example [6, p. 474]. From this the result follows.

For circumscribed geodesic spheres of $\Sigma$, we have the following:

Proposition 3.2. Let $\Sigma$ be a closed convex surface in $\mathbb{H}^{3}$ such that

$$
\lambda_{i}(x)>\operatorname{coth} a>1
$$

for $i=1,2$ and for all $x \in \Sigma$. Then there is a geodesic sphere of radius a which contains $\Sigma$ in its interior.

Since we cannot find an explicit reference for this, we will give more details of the proof. We use the idea of Andrejs Treibergs [13] to give a proof. We thank him for the idea. To show this, we need the following lemma about convex curves on $\mathbb{H}^{2}$, which is an extension of Schur's theorem for plane curves.

Lemma 3.3. Let $\alpha$ and $\beta$ be two curves in $\mathbb{H}^{2}$ with the same length $l$ parametrized by arc length. Let $\gamma$ be the geodesic from $\alpha(0)$ to $\alpha(l)$ and let $\sigma$ be the geodesic from $\beta(0)$ to $\beta(l)$. Suppose $\alpha$ and $\gamma$ bound a geodesically convex region, and $\beta, \sigma$ bound a geodesically convex region. Suppose the geodesic curvature $k$ of $\alpha$ is larger than the geodesic curvature $\tilde{k}$ of $\beta$, which are assumed to be positive. Then the length of $\gamma$ is less than the length of $\sigma$.

Proof. Let us use the right half-plane model for $\mathbb{H}^{2}$ :

$$
\mathbb{H}^{2}=\left\{(x, y) \in \mathbb{R}^{2} \mid x>0\right\}
$$

with metric $d s^{2}=\frac{d x^{2}+d y^{2}}{x^{2}}$. We may assume that $\gamma$ is given by $\gamma(t)=(t, c), a \leq t \leq b$ and $c$ is a constant. We also assume that $\alpha$ is below $\gamma$. That is, if $\alpha(s)=(x(s), y(s))$, then $y(s) \leq c$. We may assume that $\alpha$ touches the geodesic $\left(t, c^{\prime}\right)$ for some $c^{\prime}$ at $\alpha\left(s_{0}\right)$ and some $0<s_{0}<l$. Then $\alpha$ lies between the geodesics $y=c$ and $y=c^{\prime}$. Move $\beta$ such that $\beta\left(s_{0}\right)=\alpha\left(s_{0}\right), \beta\left(s_{0}\right)$ touches $y=c^{\prime}$ at $\beta\left(s_{0}\right)$ and $\beta$ lies above $y=c^{\prime}$; i.e. $\beta$ is in the region $y \geq c^{\prime}$. 
Let $\alpha(s)=(x(s), y(s))$ and $\beta(s)=(\widetilde{x}(s), \widetilde{y}(s))$. Let $\theta(s)$ be the oriented angle from the tangent of the geodesic $t \mapsto(t, y(s))$ to $\alpha^{\prime}(s)$. Define $\widetilde{\theta}(s)$ for $\beta$ similarly so that $\theta\left(s_{0}\right)=\widetilde{\theta}\left(s_{0}\right)=0$.

Note that for any $l>s>s^{\prime}>s_{0}, y(s) \neq y\left(s^{\prime}\right)$; otherwise the curve $t \mapsto(t, y(s))$ is a part of $\alpha$ which is a geodesic. This is impossible, because $k>0$. Hence $y$ is increasing in $\left(s_{0}, l\right)$. Since

$$
x^{\prime}=x \cos \theta, \quad y^{\prime}=x \sin \theta,
$$

$\sin \theta \geq 0$. But for $s_{0}<s<l$, if $\sin \theta(s)=0$, then the geodesic $t \mapsto(t, y(s))$ is tangent to $\alpha$, which is impossible because of the convexity of the region bounded by $\alpha$ and $\gamma$. So $\sin \theta>0$ there.

On the other hand, we have [3, p. 253]:

$$
k=-\sin \theta+\theta^{\prime} .
$$

Hence $0<\theta \leq \pi$ on $\left(s_{0}, l\right)$. Similarly, we have

$$
\widetilde{k}=-\sin \widetilde{\theta}+\widetilde{\theta}^{\prime}
$$

Since $k>\widetilde{k}$ and $\theta\left(s_{0}\right)=\widetilde{\theta}\left(s_{0}\right)=0$, so for $s>s_{0}$ near $s_{0}, \theta(s)>\widetilde{\theta}(s)$. Suppose there is a first $l>s_{1}>s_{0}$ such that $\theta\left(s_{1}\right)=\widetilde{\theta}\left(s_{1}\right)$. Then at $s_{1}$,

$$
k-\widetilde{k}=\theta^{\prime}\left(s_{1}\right)-\widetilde{\theta}^{\prime}\left(s_{1}\right) \leq 0 .
$$

This is impossible. Hence $0 \leq \widetilde{\theta}(s) \leq \theta(s) \leq \pi$ in $\left(s_{0}, l\right)$.

Now

$$
\log x(l)-\log x\left(s_{0}\right)=\int_{s_{0}}^{l} \frac{x^{\prime}}{x} d s=\int_{s_{0}}^{l} \cos \theta(s) d s
$$

and

$$
\log \widetilde{x}(l)-\log \widetilde{x}\left(s_{0}\right)=\int_{s_{0}}^{l} \frac{\widetilde{x}^{\prime}}{\widetilde{x}} d s=\int_{s_{0}}^{l} \cos \widetilde{\theta}(s) d s .
$$

Hence $\log \widetilde{x}(l) \geq \log x(l)=\log b$. Similarly, one can prove that $\log \widetilde{x}(0) \leq \log x(0)=$ $\log c$. In particular, $\widetilde{x}(0)<\widetilde{x}(l)$. Now the length $L(\gamma)$ of $\gamma$ is $\log b-\log c$. Hence $L(\gamma) \leq \log \widetilde{x}(l)-\log \widetilde{x}(0)$.

We claim that $\log \widetilde{x}(l)-\log \widetilde{x}(0) \leq L(\sigma)$. We may assume $\widetilde{y}(0)<\widetilde{y}(l)$. Then $\log \widetilde{x}(l)-\log \widetilde{x}(0)$ is the length of the geodesic $(t, \widetilde{y}(l)), \widetilde{x}(0)<t<\widetilde{x}(l)$. Then by the sine law in $\mathbb{H}^{2}$, we conclude that the claim is true. This completes the proof of the lemma.

Lemma 3.4. Let $\alpha$ be a closed geodesically convex curve in $\mathbb{H}^{2}$ with geodesic curvature $k_{\alpha}>r>0$. Let $\beta$ be a geodesic circle with geodesic curvature $r$. Suppose $\alpha$ and $\beta$ are tangent at $p$ such that $\alpha$ and $\beta$ lie on the same side of the geodesic through $p$ and tangent to $\alpha$ and $\beta$. Then $\alpha$ will lie inside $\beta$.

Proof. We use the disk model for $\mathbb{H}^{2}$. We may assume that $\beta$ is a Euclidean circle with center at the origin and with radius $a>0$, say. We may also assume that $p=(0,-a)$ and $\beta$ is parametrized by $(a \cos \theta, a \sin \theta),-\pi \leq \theta \leq \pi$. It is easy to see that $(\theta)$ is outside $\alpha$ near $p$, for $\theta \in\left(-\frac{\pi}{2}-\theta_{0},-\frac{\pi}{2}+\theta_{0}\right)=I$ for some $\theta_{0}>0$. Suppose the lemma is not true. Then $\beta$ will intersect $\alpha$ at some $\theta_{1} \notin I$. Without loss of generality, we may assume that there is $\frac{\pi}{2} \geq \theta_{1} \geq-\frac{\pi}{2}+\theta_{0}$ such that $\alpha$ and $\beta$ intersect at $q=\left(\theta_{1}\right)$ and $(\theta)$ lies strictly outside $\alpha$ for all $\theta \in\left(-\frac{\pi}{2}+\theta_{0}, \theta_{1}\right)$. Then 
the length of $\beta$ from $p$ to $q$ is strictly greater than the length of $\alpha$ from $p$ to $q$ by the Gauss-Bonnet theorem and the fact that $k_{\alpha}>r$. Then there is $\theta_{1}>\theta_{2}>-\frac{\pi}{2}+\theta_{0}$ such that the length of $\beta$ from $p$ to $u=\left(\theta_{2}\right)$ is the same as the length of $\alpha$ from $p$ to $q$. By Lemma 3.3. we conclude that $d(p, q) \leq d(p, u)$. Since $p, q, u$ are on the geodesic circle $\beta$, this is impossible by the cosine law in $\mathbb{H}^{2}$.

Proof of Proposition 3.2. Let $p \in \Sigma$. Let $S$ be the geodesic sphere with radius $a$ which is tangent to $\Sigma$ at $p$ with the same unit outward normal at $p$. Let $P$ be any normal section. That is, $P$ is the totally geodesic $\mathbb{H}^{2}$ which passes through $p$ and contains the geodesic normal to $\Sigma$ (and $S$ ) at $p$. Let $\gamma=P \cap \Sigma$ and $\beta=P \cap S$.

Since the principal curvature of $\Sigma$ is greater than $\operatorname{coth} a, \gamma$ is a closed convex curve in $P$ with geodesic curvature greater than $\operatorname{coth} a . \quad \beta$ is a geodesic circle of radius $a$ in $P$. By Lemma 3.4, $\gamma$ lies inside $\beta$ and hence is inside $S$. Since $P$ is an arbitrary normal section, the result follows.

\section{Normalized embedding of $\left(S_{r}, \gamma_{r}\right)$}

Let $\left(M^{3}, g\right)$ be an AH manifold satisfying Assumption A. Let $\left(S_{r}, \gamma_{r}\right)$ be as in Lemma 2.1. The isometric embedding of $\left(S_{r}, \gamma_{r}\right)$ in $\mathbb{H}^{3}$ is unique up to an isometry of $\mathbb{H}^{3}$. In order to prove the main results, we have to normalize the embedding. As a first step, using Lemmas 2.1 and 2.5. we can apply Propositions 3.1 and 3.2 to obtain the following:

Lemma 4.1. With the above assumptions and notation, we can find a positive constant $C$ such that for each small $r$, if $\Sigma_{r}$ is the isometric embedding of $\left(S_{r}, \gamma_{r}\right)$ in $\mathbb{H}^{3}$, then there exist geodesic balls $B_{\text {in }}$ and $B_{\text {out }}$ with the same center and radii $\rho_{\text {in }}$ and $\rho_{\text {out }}$ respectively such that $B_{\text {in }}$ is in the interior of $\Sigma_{r}, B_{\text {out }}$ contains $\Sigma_{r}$, and $\rho_{\text {in }}, \rho_{\text {out }}$ satisfy:

$$
\rho_{\text {in }} \geq \sigma-C r^{3}, \rho_{\text {out }} \leq \sigma+C r^{3},
$$

where $\sigma=\sigma(r)>0$ is given by $\sinh \sigma=1 / \sinh r$.

Proof. Let $r$ be a fixed small number. Let $\lambda_{j}(x)$ be the principal curvatures of $\Sigma_{r}$. By Lemmas 2.1 2.5 and the Gauss equation, we have

$$
\lambda_{j}=\cosh r+O\left(r^{5}\right) .
$$

Let $\operatorname{coth} \rho=\lambda_{j}$. Then

$$
\begin{aligned}
\rho=\frac{1}{2} \log \left(\frac{\lambda_{j}+1}{\lambda_{j}-1}\right)=\frac{1}{2} \log \left(\frac{\cosh r+1+O\left(r^{5}\right)}{\cosh r-1+O\left(r^{5}\right)}\right) & =\frac{1}{2} \log \left(\frac{\cosh r+1}{\cosh r-1}\right)+O\left(r^{3}\right) \\
& =\sigma+O\left(r^{3}\right) .
\end{aligned}
$$

From this and Propositions 3.1 and 3.2 the result follows.

By Lemma 4.1, the first normalization of the embedding is to normalize such that the center of the geodesic balls in Lemma 4.1 is at a fixed point $o \in \mathbb{H}^{3}$. We will use geodesic polar coordinates $(\sigma, y)$ with center at $o$, where $\sigma$ is the geodesic distance from $o$ and $y \in \mathbb{S}^{2}$ so that a point in $\mathbb{H}^{3}$ is of the form $\exp _{o}(\sigma y)$. The metric $g_{\mathbb{H}^{3}}$ is given by $d \sigma^{2}+\sinh ^{2} \sigma g_{0}$, where $g_{0}$ is the standard metric on $\mathbb{S}^{2}$. The isometric embedding $X^{(r)}$ is given by $X^{(r)}(x)=\exp _{o}\left(\sigma^{(r)}(x) y^{(r)}(x)\right)$. 
Lemma 4.2. With the above notation, there exists a constant $C>0$ such that for sufficiently small $r$ and $x_{1}, x_{2} \in \mathbb{S}^{2}$,

$$
\left|d_{\mathbb{S}^{2}}\left(x_{1}, x_{2}\right)-d_{\mathbb{S}^{2}}\left(y^{(r)}\left(x_{1}\right), y^{(r)}\left(x_{2}\right)\right)\right| \leq C r^{3},
$$

where $d_{\mathbb{S}^{2}}$ is the distance on $\mathbb{S}^{2}$ with respect to the standard metric.

Proof. Let $x_{1}, x_{2} \in \mathbb{S}^{2}$ and let $X^{(r)}$ be as above so that the embedded image $\Sigma_{r}$ lies between two concentric geodesic spheres $\partial B_{o}\left(R_{1}\right)$ and $\partial B_{o}\left(R_{2}\right)$ with center at $o$ and with radii $R_{1}>R_{2}$ such that $R_{i}=\sigma+O\left(r^{3}\right), i=1,2$, and $\sigma$ is given by $\sinh \sigma=\frac{1}{\sinh r}$, by Lemma 4.1. Here and below $O\left(r^{k}\right)$ will denote a quantity with absolute value bounded by $C r^{k}$ for some positive constant $C$ independent of $r$ and $x_{1}, x_{2} \in \mathbb{S}^{2}$

Let $l\left(x_{1}, x_{2}\right)$ be the intrinsic distance between $x_{1}, x_{2} \in S_{r}$ with respect to the metric $\gamma_{r}$. By the definition of an AH manifold, it can be seen that

$$
l\left(x_{1}, x_{2}\right)=\frac{1}{\sinh r} d_{\mathbb{S}^{2}}\left(x_{1}, x_{2}\right)\left(1+O\left(r^{3}\right)\right) .
$$

On the other hand, let $v_{1}, v_{2}$ be the points of intersections of $\partial B_{o}\left(R_{2}\right)$ with the geodesics from $o$ to $X^{(r)}\left(x_{1}\right)$ and $X^{(r)}\left(x_{2}\right)$ respectively. Since $X^{(r)}$ is an isometric embedding, the intrinsic distance between $X^{(r)}\left(x_{1}\right)$ and $X^{(r)}\left(x_{2}\right)$ in $\Sigma_{r}$ is equal to $l\left(x_{1}, x_{2}\right)$. Since $\Sigma_{r}$ is strictly convex in $\mathbb{H}^{3}$ by (4.2) and $R_{i}=\sigma+O\left(r^{3}\right)$, we have

$$
l\left(x_{1}, x_{2}\right) \leq d_{\partial B_{o}\left(R_{2}\right)}\left(v_{1}, v_{2}\right)+O\left(r^{3}\right)
$$

because $l\left(x_{1}, x_{2}\right)$ is the minimum of lengths of curves in $\mathbb{H}^{3}$ outside $\Sigma_{r}$ which join $X^{(r)}\left(x_{1}\right)$ and $X^{(r)}\left(x_{2}\right)$. Here $d_{\partial B_{o}\left(R_{2}\right)}$ is the intrinsic distance function on $\partial B_{o}\left(R_{2}\right)$. So we have

$$
l\left(x_{1}, x_{2}\right) \leq \sinh \sigma d_{\mathbb{S}^{2}}\left(y^{(r)}\left(x_{1}\right), y^{(r)}\left(x_{2}\right)\right)+O\left(r^{2}\right) .
$$

Using the fact that $\partial B_{o}\left(R_{1}\right)$ is also strictly convex, similarly we have

$$
l\left(x_{1}, x_{2}\right) \geq \sinh \sigma d_{\mathbb{S}^{2}}\left(y^{(r)}\left(x_{1}\right), y^{(r)}\left(x_{2}\right)\right)+O\left(r^{2}\right) .
$$

Combining these two inequalities we have

$$
l\left(x_{1}, x_{2}\right)=\sinh \sigma d_{\mathbb{S}^{2}}\left(y^{(r)}\left(x_{1}\right), y^{(r)}\left(x_{2}\right)\right)+O\left(r^{2}\right) .
$$

By (4.3), (4.4) and the fact that $\sinh \sigma=\frac{1}{\sinh r}$, the result follows.

Let $X^{(r)}$ be the isometric embeddings normalized as above.

Lemma 4.3. With the above notation, by composing $X^{(r)}$ with isometries of $\mathbb{H}^{3}$ fixing $o$, and the resulting isometric embeddings still denoted by $X^{(r)}$, we have

$$
\lim _{r \rightarrow 0} y^{(r)}(x)=x, \quad x \in \mathbb{S}^{2} .
$$

The convergence is uniform in $x$.

Proof. For $x=\left(x^{1}, x^{2}, x^{3}\right) \in \mathbb{S}^{2}, \sum_{i}\left(x^{i}\right)^{2}=1$. Let $e_{i}$ be the standard basis in $\mathbb{R}^{3}$. By composing with isometries of $\mathbb{H}^{3}$ fixing $o$, we may assume that

$$
y^{(r)}\left(e_{1}\right)=e_{1}, y^{(r)}\left(e_{2}\right) \in\left\{x^{3}=0, x^{2} \geq 0\right\}, y^{(r)}\left(e_{3}\right) \in\left\{x^{3} \geq 0\right\}
$$

for all $r$. By Lemma 4.2 .

$$
d_{\mathbb{S}^{2}}\left(y^{r}\left(e_{2}\right), e_{1}\right)=d_{\mathbb{S}^{2}}\left(y^{r}\left(e_{2}\right), y^{(r)}\left(e_{1}\right)\right)=d_{\mathbb{S}^{2}}\left(e_{2}, e_{1}\right)+O\left(r^{3}\right) .
$$


By (4.5), we can conclude that $\lim _{r \rightarrow 0} y^{(r)}\left(e_{2}\right)=e_{2}$. Let $r_{n} \rightarrow 0$ be such that $y^{\left(r_{n}\right)}\left(e_{3}\right) \rightarrow a=\left(a^{1}, a^{2}, a^{3}\right)$ with $a^{3} \geq 0$. By Lemma4.2 again, we have $d_{\mathbb{S}^{2}}\left(e_{1}, a\right)=$ $d_{\mathbb{S}^{2}}\left(e_{2}, a\right)=\frac{\pi}{2}$ and hence $a=e_{3}$. That is, we have

$$
\lim _{r \rightarrow 0} y^{(r)}\left(e_{i}\right)=e_{i}, \text { for } 1 \leq i \leq 3 .
$$

Now for any $x \in \mathbb{S}^{2}, r_{n} \rightarrow 0$ such that $\lim _{n \rightarrow \infty} y^{\left(r_{n}\right)}(x)=b$. Then by (4.6) and Lemma 4.2, we have $d_{\mathbb{S}^{2}}\left(e_{i}, b\right)=d_{\mathbb{S}^{2}}\left(e_{i}, x\right)$ for $1 \leq i \leq 3$. Hence $b=x$ and so $\lim _{r \rightarrow 0} y^{(r)}(x)=x$ for all $x \in \mathbb{S}^{2}$.

We claim that the convergence is uniform. Fix $x_{0} \in \mathbb{S}^{2}$ for any $\epsilon>0$. By Lemma 4.2 let $C$ be the constant in the lemma. Then for any $x \in \mathbb{S}^{2}$ with $d_{\mathbb{S}^{2}}\left(x, x_{0}\right)<\epsilon$, we have

$$
\begin{aligned}
d_{\mathbb{S}^{2}}\left(y^{(r)}(x), x\right) & \leq d_{\mathbb{S}^{2}}\left(y^{(r)}(x), y^{(r)}\left(x_{0}\right)\right)+d_{\mathbb{S}^{2}}\left(y^{(r)}\left(x_{0}\right), x_{0}\right)+d_{\mathbb{S}^{2}}\left(x_{0}, x\right) \\
& \leq 2 d_{\mathbb{S}^{2}}\left(x_{0}, x\right)+d_{\mathbb{S}^{2}}\left(y^{(r)}\left(x_{0}\right), x_{0}\right)+C r^{3} \leq 3 \epsilon
\end{aligned}
$$

provided $r$ is small enough depending only on $x_{0}$ and $\epsilon$. Since $\mathbb{S}^{2}$ is compact, this proves the claim that the convergence is uniform.

\section{Proof of Theorem 1.3}

We now prove our main results. First, we embed $\mathbb{H}^{3}$ in the $\mathbb{R}^{3,1}$ so that $\mathbb{H}^{3}=$ $\left\{\left(x^{0}, x^{1}, x^{2}, x^{3}\right) \in \mathbb{R}^{3,1}:\left(x^{0}\right)^{2}-\sum_{i=1}^{3}\left(x^{i}\right)^{2}=1, x^{0}>0\right\}$ and the fixed point $o$ in Section 4 is mapped to the point $(1,0,0,0)$.

Proof of Theorem 1.3. For $r$ small, let $X^{(r)}$ be the embedding of $\left(S_{r}, \gamma_{r}\right)$ in $\mathbb{H}^{3}$ given by Lemma 4.3. With the notation as in Section 4 when considered as an embedding of $\left(S_{r}, \gamma_{r}\right)$ in $\mathbb{R}^{3,1}, X^{(r)}$ is of the form

$$
X^{(r)}(x)=\left(\cosh \sigma^{(r)}(x), \sinh \sigma^{(r)}(x) y^{(r)}(x)\right) .
$$

Now by Corollary 2.6. Lemmas 4.1, 4.3 and Definition 1.1, as $r \rightarrow 0$,

$$
\begin{cases}H_{0}-H & =\frac{r^{3}}{2} \operatorname{tr}_{g_{0}} h+O\left(r^{4}\right) \\ \cosh \sigma^{(r)}(x) & =\operatorname{coth} r+O\left(r^{2}\right)=\frac{1}{r}+o(1), \\ \sinh \sigma^{(r)}(x) & =\frac{1}{\sinh r}+O\left(r^{2}\right)=\frac{1}{r}+o(1), \\ y^{(r)}(x) & =x+o(1), \\ d \mu_{\gamma_{r}} & =\left(\frac{1}{\sinh ^{2} r}+O\left(r^{3}\right)\right) d \mu_{g_{0}}=\left(\frac{1}{r^{2}}+o(1)\right) d \mu_{g_{0}} .\end{cases}
$$

As before, $O\left(r^{k}\right)$ represents a quantity with absolute value bounded by $C r^{k}$ with $C$ being independent of $r$ and $x$. By (5.1), (5.2), we have

$$
\begin{aligned}
& \int_{S_{r}}\left(H_{0}-H\right) X^{(r)} d \mu_{\gamma_{r}} \\
= & \int_{\mathbb{S}^{2}}\left(\left(\frac{r^{3}}{2} \operatorname{tr}_{g_{0}} h+O\left(r^{4}\right)\right)\left(\frac{1}{r}+o(1), \frac{x}{r}+o\left(\frac{1}{r}\right)\right)\left(\frac{1}{r^{2}}+o(1)\right)\right) d \mu_{g_{0}} \\
= & \frac{1}{2}\left(\int_{\mathbb{S}^{2}} \operatorname{tr}_{g_{0}}(h) d \mu_{g_{0}}, \int_{\mathbb{S}^{2}} \operatorname{tr}_{g_{0}}(h) x d \mu_{g_{0}}\right)+o(1) .
\end{aligned}
$$

From this the theorem follows. 


\section{ACKNOWLEDGMENTS}

The first author would like to thank Chit-Yu Ng, and the second author would like to thank Ralph Howard, Yuguang Shi and Andrejs Treibergs for useful and stimulating discussions.

\section{REFERENCES}

1. L. Andersson and M. Dahl, Scalar curvature rigidity for asymptotically locally hyperbolic manifolds, Annals of Global Analysis and Geometry 16 (1998), no. 1, 1-27. MR.1616570 (99d:53035)

2. P. Chruściel and M. Herzlich, The mass of asymptotically hyperbolic Riemannian manifolds, Pacific J. Math. 212 (2003), no. 2, 231-264. MR2038048 (2005d:53052)

3. M. do Carmo, Differential geometry of curves and surfaces, Prentice Hall, 1976. MR 0394451 (52:15253)

4. X.-Q. Fan and K.-K. Kwong, The Brown-York mass of revolution surfaces in asymptotically Schwarzschild manifolds, J. Geom. Anal. 21 (2011), no. 3, 527-542. MR2810842

5. X.-Q. Fan, Y. G. Shi and L.-F. Tam, Large-sphere and small-sphere limits of the Brown-York mass, Commun. Anal. Geom. 17 (2009), no. 1, 37-72. MR2495833 (2010e:53132)

6. R. Howard, Blaschke's rolling theorem for manifolds with boundary, Manuscripta Mathematica 99 (1999), no. 4, 471-483. MR.1713810 (2000i:53047)

7. Y. Li and G. Weinstein, A priori bounds for co-dimension one isometric embeddings, American Journal of Mathematics 121 (1999), no. 5, 945-965. MR.1713298 (2000g:53071)

8. P. Miao, Positive mass theorem on manifolds admitting corners along a hypersurface, Adv. Theor. Math. Phys. 6 (2002), no. 6, 1163-1182 (2003). MR1982695 (2005a:53065)

9. A. Pogorelov, Some results on surface theory in the large, Advances in Math. 1 (1964), no. 2, 191-264. MR0178438 (31:2695)

10. Y. G. Shi and L.-F. Tam, Positive mass theorem and the boundary behaviors of compact manifolds with nonnegative scalar curvature, J. Differential Geom. 62 (2002), 79-125. MR1987378 (2005b:53046)

11. Y. G. Shi and L.-F. Tam, Rigidity of compact manifolds and positivity of quasi-local mass, Classical Quantum Gravity 24 (2007), no. 9, 2357-2366. MR2320089(2008e:53153)

12. Y. G. Shi, G. Wang and J. Wu, On the behavior of quasi-local mass at the infinity along nearly round surfaces, Ann. Global Anal. Geom. 36 (2009), no. 4, 419-441. MR2562923 (2010m:53112)

13. A. Treibergs, Private communications.

14. X. D. Wang, The mass of asymptotically hyperbolic manifolds, J. Differential Geom. 57 (2001), no. 2, 273-299. MR1879228 (2003c:53044)

15. M. T. Wang and S. T. Yau, A generalization of Liu-Yau's quasi-local mass, Comm. Anal. Geom. 15 (2007), no. 2, 249-282. MR2344323(2008h:53046)

16. X. Zhang, A definition of total energy-momenta and the positive mass theorem on asymptotically hyperbolic 3-manifolds. I, Comm. Math. Phys. 249 (2004), no. 3, 529-548. MR2084006 (2006a:83012)

The Institute of Mathematical Sciences and Department of Mathematics, The Chinese University of Hong Kong, Shatin, Hong Kong, People' Republic of China

E-mail address: kkkwong@math.cuhk.edu.hk

Current address: School of Mathematical Sciences, Monash University, Victoria 3800, Australia

E-mail address: kwok-kun.kwong@monash.edu

The Institute of Mathematical Sciences and Department of Mathematics, The Chinese University of Hong Kong, Shatin, Hong Kong, People's Republic of China

E-mail address: lftam@math.cuhk.edu.hk 